\title{
Formación en sistemas de información electrónica y nuevos documentos
}

\author{
Francisco Javier Martínez Méndez \\ Grupo de investigación en Tecnologías de la Información \\ Departamento de Información y Documentación \\ Universidad de Murcia
}

\subsection{Resumen}

La Sociedad de la Información está incorporando nuevos roles profesionales dentro del campo del mercado de la información. Englobado dentro de un contínuo y progresivo proceso, los gestores de sistemas de información van adaptando sus tareas incorporando a ellas el uso de la Tecnología de la Información en el desarrollo de sus actividades. Este proceso está configurando un nuevo contexto, un espacio multimedia que fuerza la adaptación de los curricula de los futuros profesionales de la información con la idea de satisfacer las necesidades de sus usuarios. Este nuevo profesional podría concebirse como un Ingeniero de la Información, capaz de diseñar sistemas de información interactivos y multimedia. (Autor)

Palabras clave: Tecnología de la Información. Sociedad de la Información. Multimedia. Gestión de sistemas de información. Ingeniería de la Información.

\subsection{Abstract}

Information Society has become to introduce a new set of roles into the field of the Information Market. Included inside a continous and progresive process, the managers of information systems are modifying their jobs incorporating definitively the use of Information Technology. This progress is going to configure a new context, a new space characterised as a multimedia space that forces the adaption of the future professional curricula in order to satisfy the user's information needs. This new profile might be conceived as an Information Engineer, able to design multidemia and interactive information systems. (Author)

Keywords: Information Technology. Information Society. Multimedia. Information systems management. Information Engineering. 


\section{Hacia la Sociedad de la Información}

Parece un hecho universalmente aceptado el concepto de Sociedad de la Información que impera en nuestros días, destacando la calidad de recurso económico (estratégico por tanto) de la información, adoptando la misma un talante trascendental en la toma de decisiones de una organización.

Esta Sociedad de la Información se alcanzará cuando un porcentaje significativo de sus miembros tenga acceso a cualquier tipo de información, en cualquier formato (voz, textos, gráficos, imágenes fijas y vídeo), en cualquier momento y desde cualquier lugar. Este desarrollo conlleva la creación de un nиеvo espacio en el que se van a integrar los tres tipos de interacción que se dan actualmente:

- información

- comunicación

- transacciones (compras, educación, sanidad, ocio, etc.)

Hasta el momento, asistimos a la implantación de estas interacciones en espacios separados e independientes, situación que conduce a una serie de determinados avances independientes unos de otros que no parecen tener muy definida una solución de continuidad y que no vienen acompañados de sistemas organizacionales innovadores en los negocios o en las administraciones o en la promoción de servicios de valor añadido, derivados de este avance.

Tenemos muy presente la polémica derivada por la implantación en España de una serie de medios de comunicación conocidos como "plataformas digitales". En nuestra opinión, resulta extremadamente exagerada la presentación de estos servicios como la entrada de nuestro país en la Sociedad de la Información, cuando no dejan de ser meras evoluciones de la transmisión codificada de señales de TV por medio de satélite, señales que han pasado de formato analógico a formato digital; tratándose, por lo tanto, de un proceso similar y quizás de menor alcance, al que todos hemos asistido cuando cambiamos del disco de vinilo al CD. Estos sistemas empiezan a incorporar otros servicios derivados de su carácter "digital", como la posibilidad de transmisión de datos, aunque de forma incipiente y que pasan totalmente desapercibidos inmersos en la gran oferta de ocio que presentan a sus futuros clientes.

El alcance o consecución de la Sociedad de la Información conlleva la integración en un único espacio de las tres interacciones. Ello, implicará la modificación de la organización de los negocios y de las cadenas de valor añadido, apareciendo nuevas sinergias y oportunidades de negocio: surge un nuevo espacio para las oportunidades de las empresas y de los consumidores, un espacio donde cada ciudadano podrá elegir en cada momento la información que precise, la película que desee visualizar en el salón de su domicilio, recibir lecciones 
interactivas de idiomas o de pintura, comunicarse con sus amigos por medio de la videoconferencia, efectuar transacciones con su banco o caja de ahorros o encargar la compra al supermercado, ese nuevo espacio, que está por llegar, producto de la Sociedad de la Información será multimedia, para muchos conocido como el "cyberespacio" o "mundo digital".

Ese nuevo espacio provocará cambios substanciales en todo lo que nos circunda, los residentes en poblaciones alejadas de las grandes capitales podrán acceder a servicios que hoy en día tienen muy lejanos, esa cercanía también llegará a las personas con discapacidades físicas que podrán ver superados de alguna manera sus problemas de desplazamiento. En su seno se generarán nuevas formas de trabajo y la cultura y la educación se expandirá a colectivos que hasta ahora tienen complicado su alcance. En resumen, esta serie de evoluciones implicará en la práctica una revolución social, de características y proporciones similares a las producidas en la Historia.

Todo este cambio se viene concibiendo desde hace años, cada paso que se produce en el campo de las telecomunicaciones nos introduce más en ella, además, cobra especial impulso con el auspicio político proveniente de las iniciativas de Albert Gore o de Martin Bangemann (no sólo por ellos, evidentemente, sino por la totalidad de los ciudadanos que estos dos dirigentes representan); propiciando el desarrollo de las infraestructuras nacionales de información que serán el soporte de lo que se ha dado en llamar autopistas de la información; "estas infraestructuras deben disponer de los medios necesarios para garantizar que por ellas puede viajar cualquier tipo de información y en cualquier combinación, que es posible llegar a cualquier usuario con independencia de donde se encuentre y que la información puede permanecer almacenada en algunos puntos de la infraestructura para que sus usuarios puedan acceder a ella en el momento que lo deseen" (Linares, 1994, p.28).

El término infraestructura nacional de información se compone de dos partes claramente diferenciadas y que resultan de un interés muy significativo para nosotros:

a) El concepto "infraestructura nacional" nos interesa porque con similar denominación se designaron las anteriores iniciativas extendidas en otros campos en pos del progreso económico y social: el ferrocarril, las autopistas, etc.; acciones que nos inducen a reflexionar sobre la idea de que esta fusión de iniciativas concluirá en el desarrollo de ese nuevo espacio.

b) Con la presencia del vocablo "información", vislumbramos (y creemos firmemente que no nos equivocamos), que ese nuevo espacio, el espacio multimedia, será un espacio de información, más concretamente el espa- 
cio de la información y por ende, el espacio de los profesionales de la información.

Los profesionales de la información gozan de unas excelentes expectativas de futuro con la venidera configuración tecnológica, que será suficientemente flexible como para adaptarse a todas las condiciones de trabajo y a todos los ámbitos donde tradicionalmente se han desarrollado las actividades vinculadas a la información, "las aplicaciones de información fáciles de usar serán componentes críticos" (Gates, 1995, p.69).

También está claro que esta época de pleno cambio nos exigirá a todos un gran esfuerzo para afrontar en condiciones el reto que tenemos por delante. Sobre el desarrollo de esta infraestructura surgen dudas al respecto, unas, razonables y otras, producto de mentes retrógradas o de actitudes conservadoras, se discuten los posibles efectos negativos o positivos derivados de esta revolución que no parece dejar a nadie indiferente, ¿qué hacer al respecto?

Nosotros, como modesta aportación a esta infraestructura global, apostamos por iniciar, cuanto antes mejor, este proceso de cambio; y para ello debemos formarnos de forma adecuada y pertinente, para podernos convertirnos en Ingenieros de la Información.

\section{2. ¿Qué es Ingeniería de la Información?}

La Tecnología de la Información hasta ahora se ha considerado ligada con el proceso de datos en las organizaciones; datos de naturaleza económica o financiera como es el caso de la generación de apuntes contables en cuentas corrientes, el control de las máquinas y la resolución de ecuaciones matemáticas complejas o, de otro lado, se ha utilizado para el almacenamiento de datos de diversa naturaleza: catálogos de bibliotecas, ficheros de personal, cotizaciones a la Seguridad Social, etc.

La Tecnología de la Información no ha sido ampliamente utilizada para la difusión de la información a todos los niveles de la sociedad, ese campo lo cubren los medios de comunicación social (prensa, radio y TV). Si en algunas áreas, esto no resulta importante, podemos encontrar una amplia temática donde resulte de interés: exposición de resultados estadísticos, diseño asistido por ordenador, bases de datos, educación interactiva, ocio, etc. Nos hallamos ubicados en una nueva fase donde la transferencia y la consulta de la información se ha constituido en la principal actividad de la tecnología informática. En nuestra disciplina, la distribución de información en soporte CD-ROM puede considerarse como una primera etapa hacia la sustitución del papel por formatos puramente electrónicos, casi puede considerarse la génesis originaria de nuestro caminar hacia el destino 
final: el espacio multimedia; este primer empuje se ha visto respaldado y enriquecido gracias a Internet, paradigma de todo este cambio.

El valor añadido que dirige este movimiento es la capacidad de reutilización de la información en formato electrónico, en formas y maneras imposibles con la información escrita. Al nivel mas simple, nos encontramos con la posibilidad de insertar segmentos de documentos dentro de otro documento (por medio de un editor de texto), e incluso, aumentando el nivel de complejidad podemos llegar a establecer asociaciones entre documentos (como es el caso de los Sistemas de Hipertexto, cuya expresión mas reciente y completa la encontramos en el sistema World Wide Web).

Otros ejemplos de tecnología punta, susceptibles de incorporarse a las actividades de los profesionales de la información son los desarrollos en sistemas de videoconferencia y sistemas informáticos para trabajo cooperativo; aportaciones novedosas que permiten a un equipo de trabajo desarrollar una serie de tareas de forma simultánea desde ubicaciones remotas.

El desarrollo de los sistemas multimedia ha modificado las estructuras tradicionales en el diseño de bases de datos, inadecuadas para gestionar objetos multimedia. La arquitectura que subyace en los sistemas gestores de bases de datos tradicionales no podía soportar la gestión de documentos que incluyeran información no textual. Estamos ante un cambio de magnitudes comparables como las existentes entre el microfilm o el CD-ROM, "los documentos almacenados digitalmente del futuro incluirán imágenes, audio, instrucciones de programación para hacer posible la interactividad y la animación, o una combinación de éstos y otros elementos, estos documentos permitirán exploraciones interactivas, reemplazarán a muchos de los impresos en papel porque podrán ayudarnos de muchas maneras" (Gates, 1995, p.111).

A nivel hardware, la tecnología presenta continuos avances en el desarrollo de estaciones de trabajo multimedia, que, conectadas al conjunto de redes de transmisión de datos de banda "muy ancha", permitirán la configuración de las autopistas de la información, esqueleto del futuro espacio multimedia, donde se producirá la mayor cantidad de difusión de información que jamás ha existido y se multiplicarán de forma exponencial las posibilidades de acceso a los documentos por parte de los usuarios.

La Ingeniería de la Información es para nosotros, el campo de actividad resultante del impacto de la Tecnología de la Información sobre la gestión de los documentos en cualquier entorno organizacional, ésta se puede considerar como una extensión lógica de las tareas desarrolladas tradicionalmente en la gestión de la información. Se encuentra dirigida a la resolución adecuada de los problemas que presenta la integración de sistemas de múltiple naturaleza. 
Para ello hace falta la aplicación de técnicas de información y tecnologías de comunicaciones para lograr una mayor facilidad de uso (usability) de información electrónica en todas sus formas, incluyendo la multimedia, tal como se plasmó en las pasadas jornadas "EuroIEMasters" celebradas el pasado verano en Palma de Mallorca con la presencia de investigadores de todo el mundo. La Ingeniería de la Información abarca la totalidad del ciclo informativo: creación, difusión y utilización. Estas tres etapas se encuentran evidentemente interrelacionadas, dependiendo unas de otras. El diseño de productos y servicios de información debe tener en cuenta el entorno donde se desarrolla la actividad del usuario, y al mismo tiempo, el contenido de la información debe ser adaptable y portable a nuevos sistemas que pudieran surgir fruto del desarrollo tecnológico.

El objetivo de la Ingeniería de la Información es permitir un más fácil y selectivo acceso a los datos, junto a proporcionar una mejor interacción con los sistemas informáticos. Los proyectos de investigación que se dirigen a incrementar el valor de la información, presentan tres líneas principales: Edición electrónica, Difusión de información y Sistemas de recuperación de información, y en todos ellos aparece la tecnología multimedia.

La Edición Electrónica cubre el diseño y creación de productos informativos, de animación, de ocio, de formación que contribuyan a alcanzar la Sociedad de la Información.

Los procesos de Difusión de Información incluyen servicios de ayuda al usuario para localizar información, integración de diferentes formatos de datos en servicios centralizados o distribuidos y la tasación económica de cada servicio; para que esta actividad pueda llevarse a cabo no debemos olvidar el medio para su realización: las redes de transmisión de datos, que aparecen ligadas íntimamente al espacio multimedia.

Por último, la Recuperación de Información por parte del usuario, incluye el desarrollo de métodos que le faciliten el acceso a la información requerida, su consulta y el proceso local de los datos dentro de su propio entorno operativo; además, esta recuperación debe estar orientada a todo tipo de usuarios, personas de diferente nivel cultural y capacidades, que deberán tener acceso a esta Sociedad de la Información, de manera que la misma garantice los principios de igualdad y de libre participación, dentro de una óptica democrática globalizadora; de hecho "para que estas informaciones sean útiles a sus usuarios habrán tenido que ser previamente transformadas en aplicaciones apropiadas para los diferentes segmentos del mercado" (Linares, 1994, p.28).

El producto de estas actividades será el espacio multimedia, donde los nuevos sistemas de acceso a la información permitirán al usuario final recuperar ingentes volúmenes de información; grandes cantidades de datos que no siempre 
le pueden resultar útiles e interesantes o bien, ante tal magnitud no puedan discernir la información pertinentemente de la que no lo es. Tenemos el ejemplo de las técnicas navegacionales de recuperación de información en Internet, los usuarios acceden a múltiples fuentes de información de forma aleatoria y casi siempre, irrepetible. El usuario sufre la problemática derivada del desbordamiento cognoscitivo (o sobrecarga de la información), y de la desubicación en el "hiperespacio". Para corregir el problema de la sobrecarga, los usuarios deberán disponer de la presencia de manuales de ayuda, guías y repertorios de fuentes de información clasificados por materias, y otra amplia serie de utilidades que les orienten en su localización de la información. También será necesario unificar las metodologías de desarrollo de sistemas de información, para favorecer la tarea de recuperación de información.

Estos inconvenientes son utilizados en contra del avance por parte de detractores pero "sin embargo, la sobrecarga de información no es específica de la autopista y no tendría por qué ser un problema. Nosotros manejamos ya increíbles cantidades de información a través de una extensa infraestructura que ha evolucionado para ayudarnos a ser selectivos: todo lo procedente de los catálogos de las bibliotecas hasta las revistas de la película, o a las páginas amarillas, o a las recomendaciones efectuadas por los amigos. Cuando alguien se preocupe por este problema, pregúntele cómo elige lo que lee. Cuando vamos a una librería no nos preocupamos por leer todos los libros que hay en ella. Deambulamos por ellas sin leer todo porque hay ayudas para la navegación que nos indican cuál es la información de interés para ayudarnos a encontrar el material impreso que deseamos. Estos indicadores incluyen el rincón de prensa, el sistema de clasificación sistemática en las bibliotecas, y los catálogos de libros en la tienda local de periódicos. En la autopista la tecnología y los servicios editoriales se combinarán para ofrecer un cierto numero de modos de ayudarnos a encontrar información" (Gates, 1995, p.79).

¿Quién pone a disposición de los ciudadanos estos medios en la actualidad?, la respuesta es fácil y no admite lugar a duda, el profesional de la información, pues bien, la misma claridad y contundencia debemos manifestar al reivindicar para el profesional de la información la responsabilidad de planificar, diseñar, desarrollar, gestionar y difundir este amplio conjunto de tareas en el futuro espacio multimedia, como evolución lógica del desempeño de las actividades tradicionales, más aún cuando los informáticos o ingenieros dirigen sus actividades en otra línea no excesivamente orientadas a la difusión de la información. Por ello, el futuro profesional debe constituirse en un "Ingeniero de la Información" o "Tecnólogo de la Información", capaz de establecer las directrices principales de una comedida utilización de los vastos recursos informativos que estarán a nuestro alcance, eludiendo así uno de los problemas que más se intuyen hoy en día: la 
infrautilización de los recursos tecnológicos en una sociedad, que podría verse abocada al retraso y subdesarrollo tanto social como estructural.

\section{3. ¿Cómo llegaremos al espacio multimedia?}

El alcance de este espacio no será sencillo ni breve. En primer lugar debemos superar la percepción de que ya estamos en él como muchos autores y medios de comunicación social preconizan. Para poder afirmar esto con rigor científico debemos dejar transcurrir algunos años a fin de que los datos estadísticos emanados de nuestra sociedad lo certifiquen de manera clara e irrevocable.

Además, este nuevo espacio debe nacer con la pretensión de instrumento para toda la sociedad, por lo que debe extenderse y alcanzar a todos los elementos participantes en la misma, y esta situación no se produce en la actualidad, donde en las diversas partes de una misma región o comunidad autónoma se presentan grandes diferencias estructurales, y nos referimos sólo a países desarrollados, sin extender nuestra reflexión a la gran mayoría de las naciones del mundo que bastante tienen con subsistir día a día sin entrar en este tipo de disquisiciones, que a ellos les pueden parecer meramente triviales, la sociedad de la información transformará profundamente la vida diaria y el tiempo de ocio, fomentará nuevas formas de desarrollo urbano y rural y mejorará la calidad de los sistemas educativo y sanitario. No obstante, la difusión acelerada de las nuevas tecnologías puede provocar también rechazo y aislamiento

Una premisa fundamental para llegar a este espacio ya la hemos introducido anteriormente, la responsabilidad del mismo debe ser compartida entre las administraciones públicas, las empresas privadas y los ciudadanos.

A las administraciones públicas les corresponderá el auspicio, la incentivación, la coordinación y la legislación relacionada con el espacio multimedia, con el único objetivo de favorecer el desarrollo del mismo en pos de la consecución del bienestar de los ciudadanos, sin injerencias ni cortapisas de ningún tipo y con ausencia total de normas y disposiciones que lo entorpezcan; siguiendo la idea expresada por la Comisión de la Unión Europea: "la Comisión estudiará el modo de impulsar la creación de condiciones favorables para los proveedores de información de manera que puedan adaptar sus conocimientos y productos al entorno cambiante y que fomenten su mayor uso".

También corresponde a las administraciones públicas la creación o adaptación, según el caso, de las normas destinadas a preservar derechos fundamentales de todos los ciudadanos como pueden ser el libre acceso a la información de las instituciones públicas, la intimidad de los datos de cada ciudadano o la pro piedad intelectual o industrial. La transformación del espacio provocará rápidas obsolescencias de las normas, por lo que las administraciones deberán ser ágiles. 
A la iniciativa privada se le debe exigir que afronte este reto con plenas garantías de consecución, que configuren un espacio accesible para todos los ciudadanos y que no vean en el mismo una fuente de especulación productora de fáciles y rápidos beneficios o un espacio destinado al establecimiento de monopolios u oligopolios que cierren la actividad a otras empresas, no se puede exigir a las administraciones públicas la liberalización total del sector para luego apoderarse de él, el mercado de la información del espacio multimedia debe ser un mercado abierto, donde se garantice el libre acceso en régimen de competencia de todos los agentes interesados.

A los ciudadanos se les debe pedir que exijan toda esta serie de cambios tanto a los estamentos públicos y privados, también se les debe exigir un uso adecuado de toda la tecnología que se pondrá a su alcance, tanto desde una óptica legal como social, evitando que usos contraproducentes de la infraestructura perjudiquen y retrasen el desarrollo del espacio multimedia.

La coordinación de las actividades a desarrollar por parte de las entidades involucradas adquiere un rol de vital importancia, el alto nivel de inversiones y de capacitaciones requerido debe desterrar la idea de desarrollos parciales, que no serían sino simples establecimientos efímeros. Los agentes llamados a formar parte de este mercado son los operadores de servicios de telecomunicaciones, los fabricantes de equipos de telecomunicaciones, las empresas informáticas y las compañías de medios (que es donde debemos hallar nuestro hábitat). La sinergia de las actividades de estos agentes permitirá la superación de los inconvenientes que se nos presentan, "el papel de los sectores públicos y privados, la interoperabilidad global, el acceso universal, la evolución de las aplicaciones, la privacidad, la seguridad, la propiedad intelectual y la estructura regulatoria" (Linares, 1994, p.29). Estos agentes deben explorar toda serie de oportunidades, experimentar con nuevos desarrollos y servicios y deben buscar la colaboración con otros socios tanto en el ámbito nacional, como internacional, con la finalidad de optimar los esfuerzos de todos y poner sus servicios a disposición de los ciudadanos en el menor tiempo posible.

Los servicios que se ofrecerán en el espacio multimedia basados en las infraestructuras nacionales de información, precisan de la utilización de tecnologías muy variadas que permitan la realización de los mismos. Los agentes participantes en esta actividad actuarán en cualquiera de estas fases: la creación de programas o contenidos (es decir la elaboración del documento); su formato de presentación a los clientes (podemos asimilar este concepto a la elección del soporte del documento), y por último el proceso de recepción por los usuarios (el marketing de la información, aspecto vital para las instituciones documentales modernas). 
El término "tecnología", no debe considerarse como una utilización práctica de sistemas instrumentales, como puede ser el caso de los ordenadores y de los sistemas informáticos que corren sobre ellos, tecnología es algo más: "la tecnología también, no lo olvidemos: es la formación y son las ideas que se enseñan, la forma de hacer, lo que en cierto modo marcará su quehacer profesional" (Maté, 1993) (1)

De cómo apliquen esta tecnología los profesionales de la información, actuando de forma clara e inequívoca, marcándose una línea general de actuación, dependerán que se los personalice y distinga de otro tipo de profesional. Su línea de actuación se mantendrá en "la confluencia de las tecnologías informática (por ejemplo: proceso de datos, gestión de sistemas de información, sistemas de ayuda en la toma de decisiones y sistemas expertos); ofimática (microfilms, fotocopias); y tecnología de telecomunicaciones (centralitas PBX's, teleconferencia, videoconferencia)" (Ang ; Pavri, 1994, p.122); llevando a cabo el desempeño del "conjunto de técnicas para la representación, almacenamiento, tratamiento y transmisión de la información, entendiendo a esta como una fórmula convencional para representar un hecho, noción o instrucción, válida para su interpretación y comunicación”. (Rodríguez, 1994, p.28).

La técnica (o aspecto práctico de la tecnología), no aparece sino en un plano secundario, posteriormente; una vez ha sido confirmado el modelo de uso de la tecnología con un cierto grado de precisión, deduciéndose de él posibles aplicaciones a poner en práctica y que serán válidas sobre la base de la experiencia desarrollada. El hallazgo de la electricidad es característico de una evolución semejante; el motor o la lámpara de incandescencia no aparecen sino bastante después de la realización de los primeros modelos de electrostática o electromagnetismo.

La tecnología es únicamente una porción del todo, puede afirmarse que es la parte ineludible (vital para algunos), pero no suficiente, precisa ser complementada por muchos aspectos, entre los cuales destaca la apropiada utilización de la misma, llegaremos al espacio multimedia con una oportuna y coherente planificación que propicie una visión integral e integrada del mismo, integral porque cubra todo el proceso e integrada por la participación de todos los estamentos sociales.

\section{4. ¿Qué aplicaciones se desarrollarán?}

Para que el espacio multimedia tenga lugar, junto al desarrollo tecnológico, precisaremos del desarrollo de un amplio conjunto de aplicaciones y soluciones dirigidas a los ciudadanos, soluciones que cubran sus necesidades, que generen beneficios económicos y sociales, que entretengan en el tiempo de ocio o que les 
informen. Todo ello contribuirá a mejorar la calidad de vida y a hacer para todos una sociedad más justa.

La mayoría de estas aplicaciones y soluciones serán de tipo multimedia, utilizarán voz, textos, gráficos, fotografías, y vídeo, combinados en la proporción pertinente para cada caso.

Cuando decimos que todos los ciudadanos son los futuros receptores de los productos emanados en el espacio multimedia, nos referimos a los ciudadanos que trabajan en las administraciones públicas, a los ciudadanos que desempeñan su actividad laboral en las empresas y también a los ciudadanos en sus hogares. Los productos a desarrollar serán específicos de cada uno de estos segmentos del mercado, o comunes implicando a varios de ellos, ya que las administraciones se relacionan con las empresas y están al servicio del ciudadano, por no hablar de la necesidad vital que tienen las empresas de contactar con los ciudadanos. Los medios de comunicación serán los mismos para todos, aunque la modalidad del servicio utilizado y desarrollado sobre ellos dependerá del segmento de aplicaciones en el que nos encontremos. A continuación introducimos algunos de esos segmentos:

\subsection{Aplicaciones para las Administraciones}

La interconexión por medio de redes de banda ancha de las distintas administraciones, con el objeto de:

- tramitar expedientes

- compartir bases de datos e intercambiar ficheros

- establecer comunicaciones interactivas por videoconferencia

- realización de trabajo cooperativo en pos de la agilización

Así se propiciará la agilización de los trámites, se aumentará la eficiencia y la administración adoptará un rol eficaz.

\subsection{Aplicaciones para las empresas}

Las empresas aumentarán la capacidad de sus medios de transmisión de datos para intercambiar datos a alta velocidad y ficheros de gran tamaño necesarios para grandes transacciones de actualización de precios en grandes almacenes, consultas rápidas en las redes bancarias o en las aplicaciones de diseño gráfico, que puede llegar a ser confeccionado sobre la base de desarrollos cooperativos.

Las videoconferencias y el teletrabajo presentan nuevas facilidades que evitarán y ahorrarán engorrosos desplazamientos con su consiguiente pérdida de tiempo y de actividad. 
La publicidad de los productos y servicios de las empresas entra en una fase donde el único límite lo pondrá la imaginación y la capacidad de los técnicos, la multimedia presenta una gama de posibilidades inmensa en este sector, ofreciendo prestaciones desde la posibilidad de presentaciones de producto de alta calidad, a catálogos interactivos o la telecompra, uniendo a este último aspecto la formación del usuario, que podemos realizarla por medio de cursos interactivos multimedia.

\subsection{Aplicaciones para la sanidad}

La sanidad debe favorecerse de las grandes redes de transmisión de datos que conllevará el intercambio rápido de historiales clínicos de pacientes, sus radiografías o analíticas. La videoconferencia permitirá diagnósticos hasta ahora imposibles por causa de la distancia o la incapacidad física de desplazamiento.

\subsection{Aplicaciones para la educación y la cultura}

La multimedia empieza a encontrar su hueco en este campo en nuestros días, cada vez es más común la producción de CD-ROM o CD-I educativos o divulgativos, y algunas editoriales configuran secciones de elaboración de productos multimedia dentro de sus organigramas. La educación asistida por ordenador se potenciará enormemente y verá aumentada de forma exponencial sus posibilidades.

Decimos lo mismo de la expansión de la cultura, tendencia que ha comenzado con Internet y que continuará hasta conseguir una interconexión total de las instituciones culturales en el ámbito mundial.

\subsection{Aplicaciones para el hogar}

En este apartado encontraremos aplicaciones desarrolladas para el ocio y esparcimiento de los ciudadanos, como puede ser la famosa "televisión a la carta", junto a otro importante conjunto de aplicaciones, mucho más solidario aunque menos conocido porque se dirige a un número menor de público como pueden ser las destinadas a personas con discapacidades o personas ancianas que pueden ser atendidas en sus propios domicilios.

El primer grupo es el sector que está propiciando las grandes inversiones financieras que nos llevan a este espacio multimedia: el gran público, es decir, nosotros. Los servicios que estarán a nuestra disposición los encontraremos en una oferta tan variada como sugerente: cine, documentales, informativos especializados, espectáculos deportivos, eventos culturales, videojuegos, recepción de mensajes, transmisión de datos, conexión a redes de ordenadores temáticas, videoconferencia, telebanco, teletrabajo, etc. ... Algunos de estos servicios (los menos), serán gratuitos y la mayor parte serán de pago, es decir, los productos del espacio multimedia tendrán un precio, una tarifa que deberemos abonar si los 
deseamos, con lo cual se producirá un cambio cuantitativo y cualitativo con respecto a hoy, la información puede dejar de ser un valor intangible, puede llegar a ser un valor tarifado.

\subsection{Teletrabajo}

Este concepto nos recuerda rápidamente el lema publicitario: "trabajar desde el lugar de tus sueños"; expresión que, quizás sea una forma onírica de compensar la necesaria carga laboral de las personas con su estancia en un lugar querido por ellos, olvidando que la mayor parte de los buenos recuerdos que nos proporcionan dichos lugares no tienen nada que ver con el trabajo, sino con las vacaciones. Bien, tras reflexionar sobre esta antítesis, la posibilidad de realizar nuestras tareas en nuestros domicilios abre la puerta al mercado laboral y genera nuevas expectativas a personas discapacitadas, con lo cual posee un marcado carácter social positivo. Además, generará nuevos perfiles profesionales y potencia la comunicación de datos entre todos los miembros de una empresa. Además, puede reducir costes en desplazamientos, mensajería e infraestructuras para las empresas, que pueden repercutir positivamente en sus beneficios.

Para finalizar con la exposición del grupo principal de aplicaciones que se desarrollarán en el futuro espacio multimedia, añadiremos que todas ellas configurarán sistemas de información interactivos, de naturaleza variada según el objeto de la misma y el público al que va dirigido, y que para su realización precisarán de los profesionales de la información.

\section{Formación de los profesionales}

Todo el amplio conjunto de considerandos anteriores nos sirve de prolegómenos para la exposición de nuestro planteamiento sobre qué contenidos deberían configurar los curricula de los profesionales de la información. Podemos establecer que hemos establecido, a un nivel básico, la materia objeto de estudio y que hemos introducido, quizás a un nivel más primario, las profesiones que desarrollarían los formados en dicha materia.

Siguiendo la lógica tradicional de un programa docente, corresponde ahora la determinación de estos contenidos y una justificación detallada de los mismos; con lo cual superaríamos con creces el objeto de este trabajo. No obstante, consideramos necesario presentar el proceso seguido para la elaboración del conjunto de puntos necesarios para la configuración de los curricula:

1. El punto de partida es la realización de una síntesis de los contenidos formativos actuales que venimos ofreciendo a nuestros alumnos, dentro de nuestro contexto docente: la Tecnología de la Información.

2. El segundo punto, que nos permite dinamizar la naturaleza de la síntesis 
anterior, es la evaluación y ponderación de estudios y proyectos internacionales en esta línea.

3. Por último, procede la incorporación de nuevos contenidos formativos que se nos presentan necesarios para un profesional de la futura Sociedad de la Información.

Obteniendo como producto final un programa completo destinado a la formación en sistemas de información electrónica y nuevos medios.

\section{Formación en Tecnología de la Información}

Hemos presentado anteriormente con suficiente claridad cuál es nuestra concepción de Tecnología de la Información, los contenidos formativos que consideramos integrantes de la misma los dividimos en los siguientes módulos:

\subsection{Conceptos básicos de la Tecnología de la Información}

- La Información y su tratamiento: Rol de la Informática en el contexto de los profesionales de la información. Representación de la información.

- Hardware: Componentes físicos del ordenador. La Unidad Central de Proceso y la Unidad de Control. La Memoria. Dispositivos periféricos: monitores, impresoras, unidades de almacenamiento de datos.

- Software: Componentes lógicos del ordenador. El Sistema Operativo. Lenguajes de Programación.

- Almacenamiento de datos: Ficheros. Bases de Datos.

- Conectividad de redes de ordenadores: Principios básicos de conectividad. Redes de Área Local, Metropolitana y Extensa. Conceptos básicos de S.O. Multiusuarios y Estructuras de Datos Distribuidas.

\subsection{Planificación de sistemas de información}

- Gestión de información: Objeto. Funciones. Elementos. Métodos. Ciclo de vida.

- Sistemas de información: Naturaleza. Elementos. Sistemas Informáticos. Diseño de un Sistema Informático. Planificación y desarrollo.

- Metodologías de diseño: Esquemas. Diagramas de flujo de datos. Metodología Métrica.

\subsection{Modelos de datos}

- Modelos de Datos: Concepto. Tipos. Modelo de Datos Entidad-Relación. Modelo de Datos Relacional.

- Álgebra de Boole: Diagramas de conjuntos. Operadores lógicos. Propiedades del álgebra de conjuntos. Su aplicación a la recuperación de 
información.

- Sistemas Gestores de Bases de Datos Relacionales: Conceptos generales. Normalización. Integridad. SQL: Lenguaje de definición de datos. SQL: Lenguaje de manipulación de datos.

- Bases de datos documentales: Concepto. Naturaleza. Tipos. Estructura y construcción de una base de datos documental.

\subsection{Sistemas de almacenamiento y recuperación de información en institu- ciones documentales}

- Automatización de las Unidades de Información: Evolución general de la automatización de las Unidades de Información. Campos de aplicación.

- Aplicaciones en Bibliotecas y Centros de Documentación: Sistemas de catalogación automatizados. Naturaleza. Gestión. Conversión retrospectiva. Cooperación bibliotecaria: sistemas y redes. Catalogación compartida entre centros. Normas para la descripción bibliográfica en soporte informático. Estructura del formato MARC. Desarrollo del formato MARC.

- Distribución de información: CD-ROM y otros dispositivos de tecnología óptica, vía redes (Internet, Compuserve, y otras..)

- Sistemas de Hipertexto: manipulación de datos multimedia

- Archivos: sistemas de hipertexto, sistemas de gestión electrónica de documentos, sistemas multimedia

- Indización asistida por ordenador.

- Telemática.

- Elementos componentes del enlace de datos.

- Modelos de referencia.

- Tipología de los sistemas.

- Protocolos de comunicaciones.

- Proceso de datos distribuido.

- Internet, Infovía. Desarrollo de sistemas de información en redes: CWIS, Intranet, otros...

Todo este cuerpo de conocimientos, en mayor o menor medida, forma parte hoy en día de los curricula de los futuros profesionales de la información. Se precisa que los mismos queden suficientemente consolidados en ellos, para poder afrontar con perspectivas de futuro el cambio que se nos avecina.

\section{7. ¿Qué faltaría?}

Es latente que nuestros profesionales de la información deben complementar Scire. 3 : 2 (jul.-dic. 1997) 11-30 
sus conocimientos ante este reto. El carácter global de todo el proceso de cambio en el que nos encontramos inmersos, sugiere la colaboración internacional para definir el conjunto de conocimientos a adquirir.

En esa línea, encontramos el trabajo desarrollado por el prestigioso profesor británico Thomas Daniel Wilson, director del departamento de Information Studies de la Universidad de Sheffield, que ha llevado a cabo una encuesta entre empresas del sector de la información dedicadas al diseño, desarrollo, edición y distribución de productos de información electrónica. Con esta encuesta, Wilson pretendía introducirnos el tipo y nivel de conocimientos y experiencia previa que serían precisos para nuevos empleados. El resultado lo presentamos a continuación:

1. Entender las necesidades de los clientes.

2. Gestión de proyectos.

3. Interfaz y diseño de interacción persona-ordenador.

4. Diseño de páginas Web.

5. Programación para multimedia.

6. Lenguajes SGML y HTML.

7. Indización y recuperación de información.

8. Gestión de servidores Web.

9. Seguridad de sistemas.

10. Trabajo en grupo por ordenador.

11. Gráficos y animación por ordenador.

12. Diseño y desarrollo de motores de búsqueda.

13. Gestión financiera y general.

14. Técnicas de producción de CD-ROM's.

15. Edición electrónica en general.

De forma paralela, Wilson realizó una encuesta entre universidades e instituciones de enseñanza superior del campo de la información y documentación, obteniendo unos resultados que debemos comentar.

Convergen ambos sectores en la importancia de los tres primeros puntos: necesidades de los clientes, gestión de proyectos y diseño de interfaces, lo que redunda aún más en la importancia de estos conceptos, en cambio, divergen de forma sorprendente en la importancia que asignan unos y otros a la creación y

mantenimiento de páginas Web y a la edición electrónica en general, donde presentan posturas muy diferentes. 
Los 15 puntos de Wilson pueden ser unificados en áreas temáticas, que presentamos en la siguiente tabla:

La primera de estas áreas, se dedica al estudio y planificación de sistemas de información. No es de extrañar su presencia, ya que este tipo de actividad cobra

\begin{tabular}{|c|c|}
\hline $\begin{array}{l}\text { Planificación de sistemas } \\
\text { informativos }\end{array}$ & $\begin{array}{l}\text { Entender las necesidades de información de los clientes. } \\
\text { Gestión de proyectos } \\
\text { Trabajo en grupo por ordenador. } \\
\text { Gestión financiera y general. }\end{array}$ \\
\hline Multimedia & $\begin{array}{l}\text { Interfaces interactivos } \\
\text { Programación para multimedia } \\
\text { Lenguajes SGML y HTML } \\
\text { Gráficos y animación por ordenador } \\
\text { Técnicas de producción de CD-ROM } \\
\text { Edición electrónica }\end{array}$ \\
\hline $\begin{array}{l}\text { Recuperación de } \\
\text { información }\end{array}$ & $\begin{array}{l}\text { Indización y recuperación } \\
\text { Diseño y desarrollo de motores de búsqueda }\end{array}$ \\
\hline $\begin{array}{l}\text { Gestión de sistemas } \\
\text { abiertos }\end{array}$ & $\begin{array}{l}\text { Gestión de servidores Web } \\
\text { Seguridad de sistemas }\end{array}$ \\
\hline
\end{tabular}

cada vez más importancia y empieza a ser considerada vital. No debería representar, no obstante, ninguna sorpresa para nosotros, que, de hecho, ya la hemos reflejado en uno de nuestros módulos anteriores.

La segunda área se presenta como un campo donde debemos profundizar, en nuestro caso particular, introduciremos en breve el estudio básico de esta serie de técnicas en nuestras acciones de formación. Resulta fácilmente deducible que, tal como hemos afirmado anteriormente, nos dirigimos al espacio multimedia, $\mathrm{y}$ como además cobra especial importancia la planificación de sistemas de información, sistemas que serán multimedias, estamos hablando de un elemento básico en la formación de los futuros profesionales.

El tercer grupo de contenidos, dirigido a las técnicas de recuperación de información es ya objeto de estudio por nuestra parte; resulta un área muy amplia y que precisa de la conformación de grupos interdisciplinares de trabajo: lingüistas, informáticos especializados en Inteligencia Artificial y Ciencias de la Computación, Matemáticos y por supuesto, especialistas en información y documentación. No es un tema nuevo, hace ya casi 50 años que surgieron las primeras iniciativas en este campo, proyectos que deben consolidarse, o al menos, producir una serie de instrumentos que nos ayuden en nuestra tarea de organizar y elaborar productos informativos en el futuro. 
Por último, la Gestión de sistemas abiertos debe dejar de ser un secreto para los profesionales de la información. Hasta ahora este campo se nos presenta como restringido a ingenieros e informáticos, dedicados a su implantación y desarrollo, que nos configuran una serie de herramientas para su posterior gestión. Si la calidad de un sistema de información depende de su proceso de planificación y diseño, y éste se deja en manos de otro tipo de profesionales, estaríamos cayendo en contradicción con lo reflejado en el área primera, y difícilmente superaríamos la concepción tradicional de un profesional de la información alejado de los sistemas informáticos.

\section{8. ¿Faltaría algo más?}

A nuestro parecer, aún deberíamos de contemplar dos áreas de interés para el futuro profesional de la información que no aparecen reflejadas en el estudio de Wilson: las telecomunicaciones y los aspectos legales derivados de la implantación de la Sociedad de la Información.

Cuando nos referimos a Telecomunicaciones, nos referimos tanto a los aspectos físicos de la tecnología como a la serie de servicios que se desarrollan o desarrollarán sobre ésta. No debemos olvidar la relación intrínseca que vamos a tener con ellos y tampoco que nuestros productos van a ir de la mano de otros diseñados para el ocio y esparcimiento de la sociedad: videojuegos, telepasatiempos, documentales o el muy nombrado en la actualidad: "pay per view". Y tampoco podemos olvidar la necesidad imperiosa de que nuestros productos no queden subsumidos en este cambio. Dentro de este área cobra especial importancia el estudio de los protocolos de transmisión de datos que facilitan la alta velocidad, caso del MTA o desarrollos posteriores, también es importante el estudio de las evoluciones de las redes metropolitanas (normas FDDI y DQDB), y de la compresión de datos para facilitar la transmisión de señales de video digital (tecnologías MPEG).

En casi todos los planes de formación y en casi todos los trabajos que reflexionan sobre ellos, nos parece detectar una escasa atención al estudio de los aspectos legales de la profesión de la información. Hoy en día, asistimos en nuestra sociedad a un debate claro sobre los derechos de transmisión de las señales de televisión, tema destacado de la actualidad social.

Reservándonos nuestra opinión sobre los procedimientos que se están llevando a cabo desde las administraciones y las empresas privadas, no conducentes en lo más mínimo a la concordia y estabilización del sector, podemos aprovecharnos de ellos, para reflexionar sobre la trascendencia que tiene sobre nuestro futuro profesional, "con la multimedia cambia la situación, por ejemplo, la combinación digital de texto, música, cuadros, fuerza a las leyes sobre informática a la convi- 
vencia con la legislación sobre música, cinematografía o espectáculos. Multimedia implica multilegia" (Hoeren, 1994. p.52).

El profesional de la formación debe conocer la legislación que de un lado le ampara, y que por otro, le indica los cauces establecidos para el desarrollo de su actividad. El cambio en esta área es imparable, el acuerdo adoptado por la Comisión de la UE y el G7 en Bruselas en 1995 así lo dictamina. Hasta ahora, los profesionales de la información podían restringir sus conocimientos a un mínimo conocimiento de la legislación sobre los derechos de autor, a la legislación sobre Patrimonio Histórico y a la Constitución de 1978, junto a algún desarrollo legal autonómico. La implantación progresiva de la Ley de Procedimiento Administrativo y de Régimen Jurídico de las Administraciones, la Ley de Ordenación y Regulación del Tratamiento Automático de Datos (LORTAD) y de la Ley de Ordenación de las Telecomunicaciones, junto al desarrollo de normativa europea relacionada con el uso nocivo de los nuevos medios de información o con la libre competencia, y no olvidemos, sobre seguridad informática donde se hace precisa la colaboración mundial, nos inducen a un nuevo marco legal que debe ser de interés para el futuro profesional de la información.

\section{Conclusiones}

De todo lo expuesto anteriormente, se infiere que surge en estos momentos, uno de los más importantes retos para los formadores: capacitar a nuestros futuros profesionales para que encuentren su hueco en la Sociedad de la Información. Así, debemos de profundizar en el estudio de todas las áreas reflejadas en lo anterior, reducir de forma paulatina estudios excesivamente cargados de planteamientos filosóficos o cuasimetafíscos sobre la teoría de la documentación, debemos evolucionar de forma ágil, porque la Universidad debe asumir su función de cabecera de un sistema formativo. Los conocimientos relacionados con las técnicas tradicionales de gestión de los documentos no deben ser obviados, sino más bien, se les debe dotar de una visión con perspectiva de futuro y de incardinarles la necesidad de sentirse vinculados en este proceso de cambio.

Para conseguirlo, debemos antes dejarnos de discusiones efímeras e intranscendentes sobre si planes de estudio de tres, cuatro, cinco o siete años, sobre pasarelas o créditos de complemento, sobre intrusismo en la profesión, sobre certificaciones o capacitaciones pseudoprofesionales, sobre la importancia de proyectos inacabados hace más de 100 años, tenemos que trabajar con vistas al futuro, que ya es presente, para algunos: para los profesionales de la información.

\section{Notas}

(1) Este enunciado del profesor Maté puede consultarse en la obra: Gamella, M.; et al. La formación universitaria en tecnologías de la información. Prospectiva de oferta y

Scire. 3 : 2 (jul.-dic. 1997) 11-30 
demanda de titulados (1992-1995). Madrid, FUNDESCO, 1993.

\section{Referencias}

Ang, J. and , Pavri, F. A Survey and Critique of the Impacts of Information Technology. // International Journal of Information Management. (14) 1994, 122-133.

Gamella, M.; et al (1993). La formación universitaria en tecnologías de la información. Prospectiva de oferta y demanda de titulados (1992-1995) // M. Gamella ; et al.. Madrid : Fundesco, 1993

Gates, Bill (1995). Camino al futuro // Bill Gates; Myhrvold N.; Rinearson P. (cols); Francisco Ortiz Chaparro (trad). Madrid : McGraw-Hill, Interamericana de España, 1995.

Hoeren, T (1994). Legal aspects of multimedia // Legal aspects of Multimedia and GIS // European Commission. Luxembourg : DG-XIII, 1994. 51-62.

Linares, Julio (1995). Autopistas inteligentes // Julio Linares, Francisco Ortiz Chaparro. Madrid : Fundesco, 1995.

Rodríguez Muñoz, J.V.(1994). Proyecto docente de Profesor Titular de Universidad en el Área de Conocimientos de Biblioteconomía y Documentación (inédito) // José Vicente Rodríguez Muñoz. Universidad de Murcia, 1994.

Sebastiá, M. (1992). De la invisibilidad a la visibilidad. Reflexiones sobre la formación de especialistas y usuarios de las Tecnologías de la Información. // Revista Española de Documentación Científica. 15 (1) 1992. 31-43. 